\title{
Evaluation of Four Genetic Variants in Han Chinese Subjects with High Myopia
}

\author{
Zimeng Ye, ${ }^{1,2}$ Huaichao Luo, ${ }^{2}$ Bo Gong, ${ }^{2,3}$ Ying Lin,,3 Ping Shuai, ${ }^{2}$ Pu Wang, \\ Changning Ye, ${ }^{3}$ Zhenglin Yang, ${ }^{2,3,4}$ Wanjun Wang, ${ }^{1}$ and $\mathrm{Yi} \mathrm{Shi}^{2,3,4}$ \\ ${ }^{1}$ College of Life Science and Engineering, Southwest Jiaotong University, 111, First Section, Northern Second Ring Road, \\ Chengdu, Sichuan 610031, China \\ ${ }^{2}$ Sichuan Provincial Key Laboratory for Human Disease Gene Study, Hospital of the University of Electronic Science and \\ Technology of China and Sichuan Provincial People's Hospital, 32 the First Ring Road West 2, Chengdu, Sichuan 610072, China \\ ${ }^{3}$ Department of Laboratory Medicine, Sichuan Provincial People's Hospital, 32 the First Ring Road West 2, \\ Chengdu, Sichuan 610072, China \\ ${ }^{4}$ School of Medicine, University of Electronic Science and Technology of China, 32 the First Ring Road West 2, \\ Chengdu, Sichuan 610072, China
}

Correspondence should be addressed to Wanjun Wang; wanjunwang@home.swjtu.edu.cn and Yi Shi; yshi@uestc.edu.cn

Received 24 September 2014; Revised 19 December 2014; Accepted 19 December 2014

Academic Editor: Edward Manche

Copyright (C) 2015 Zimeng Ye et al. This is an open access article distributed under the Creative Commons Attribution License, which permits unrestricted use, distribution, and reproduction in any medium, provided the original work is properly cited.

\begin{abstract}
High myopia is one of the leading causes of blindness worldwide. However, the exact etiology of high myopia remains unraveled despite numerous attempts of elucidation. Previous genome-wide association study (GWAS) has revealed that four single nucleotide polymorphisms (SNPs), including rs2969180, rs1652333, rs9307551, and rs7837791, were associated with high myopia in Caucasians. The present study was conducted to investigate whether these genetic variants were associated with high myopia in Han Chinese. These four SNPs were genotyped by SNaPshot method in a Han Chinese cohort composed of 827 patients with high myopia and 988 healthy controls. Among the SNPs genotyped, only rs9307551 was found to be significantly associated with high myopia in this study. Carriers of rs9307551A allele, AA, and AC genotypes had an increased risk of high myopia $(\mathrm{OR}=1.33,95 \% \mathrm{CI} 1.14-1.54$; OR $=$ $1.75,95 \%$ CI 1.28-2.38; OR $=1.59,95 \%$ CI 1.24-2.01, resp.). Interestingly, when split by gender, the association between rs 9307551 and high myopia proved to be gender-specific with significance observed only in females but not males. These findings suggested that the SNP of rs9307551 showed a gender-specific association with high myopia in the Han Chinese population. In addition, LOC100506035, a lincRNA gene, might play a crucial role in the susceptibility to high myopia.
\end{abstract}

\section{Introduction}

Myopia is a common cause of visual impairment worldwide [1], with a prevalence of $33.1 \%$ in the USA [2], $16.4 \%$ in Australia, and $26.6 \%$ in the Western Europe [3]. The prevalence of myopia in Asian populations is up to $40-70 \%$ [4-6]. In mainland China, almost $43.9 \%$ of the population suffer from myopia [7]. Low myopia (with a refractive error $\geqslant$ $-3.0 \mathrm{D}$ ) and medium myopia (with a refractive error between -6.0 and $-3.0 \mathrm{D}$ ) are primarily physiological, whereas high myopia (with a refractive error $\leqslant-6.0 \mathrm{D}$ ) is one of the leading causes of blindness in the world. High myopia (HM) is associated with pathologic ocular changes, such as myopic macular degeneration, glaucoma, and retinal detachment [810]. In China, the prevalence of HM is $4.1 \%$ [7] and is much higher in high school and university students [11].

Previous studies have indicated the involvement of genetic and environmental factors in the pathogenesis of myopia; genetic factors play a major role in the development of high myopia [12,13]. Recently, the application of genomewide association study (GWAS) has revealed several novel single nucleotide polymorphisms (SNPs) susceptible to HM in the Caucasian populations, including rs2969180, rs1652333, rs9307551, and rs7837791 [14]. Here, in order to investigate whether these SNPs were associated with high myopia in Han Chinese population, we conducted a case-control study in 
TABLE 1: Demographic and clinical characteristics of HM patients and controls in this study.

\begin{tabular}{|c|c|c|c|c|c|c|c|c|}
\hline \multirow{2}{*}{ Group } & \multirow{2}{*}{ Number } & \multirow{2}{*}{ Average age (years) } & \multicolumn{2}{|c|}{ Gender } & \multicolumn{2}{|c|}{ Refractive errors (diopter) } & \multicolumn{2}{|c|}{ Axial length (mm) } \\
\hline & & & Male & Female & OD & OS & OD & OS \\
\hline Patient & 827 & $35.30 \pm 16.55$ & 318 & 509 & $-11.34 \pm 4.65$ & $-11.04 \pm 4.49$ & $28.16 \pm 2.24$ & $27.97 \pm 2.41$ \\
\hline Control & 988 & $49.68 \pm 17.22$ & 581 & 407 & & & & \\
\hline
\end{tabular}

\pm : standard deviation; OD: right eye; OS: left eye.

a Han Chinese cohort composed of 827 unrelated individuals with high myopia and 988 unrelated normal controls.

\section{Materials and Methods}

2.1. Study Population. This study was approved by the Institutional Review Board of Sichuan Provincial People's Hospital. All participants gave their written informed consent to participate in the study. The study was performed under the principles of the Declaration of Helsinki. Patients with HM and normal matched controls were recruited at the Ophthalmology Clinic of Sichuan Provincial People's Hospital. All participants went through a standard examination protocol. In this study, the diagnosis for high myopia required the spherical equivalent less than or equal to -6.0 DS in both eyes and the axial length of the eye globe greater than or equal to $26.0 \mathrm{~mm}$. Individuals were excluded from the study if they had undergone ocular procedures that might alter refraction or if they had other symptoms besides HM. For the controls, the criteria were no evidence of disease in either eye and a spherical equivalent from -1.0 to +1.0 DS. The controls were from the same district as the cases. All of the participants in this study were Han Chinese. In total, $827 \mathrm{HM}$ patients and 988 normal controls were recruited. Demographic and clinical information of the patients and controls were listed in Table 1.

2.2. Selection of SNPs. In 2013, the Consortium of Refractive Error and Myopia (CREAM) performed a genome-wide meta-analysis of multiancestry cohorts and identified 16 new loci for refractive error in individuals of European ancestry, of which 8 were shared with Asians. Combined analysis identified 8 additional associated loci, of which 5 were shared with Asians [14]. In this study, we chose 4 of these 13 shared SNPs and planned to investigate the other 9 in future studies. The 4 SNPs we selected were rs2969180 (intronic SHISA6 gene), rs1652333 (24 kb 5' of CD55 gene), rs9307551 (33 kb $5^{\prime}$ of LOC100506035 gene), and rs7837791 (147 kb $3^{\prime}$ of TOX gene).

2.3. Genotyping. Venous blood of each subject was drawn and collected in an EDTA-containing tube. Genomic DNA was extracted from the blood using a Gentra Puregene Blood DNA kit (Gentra, Minneapolis, MN). SNP genotyping was conducted by the dye terminator-based SNaPshot method, with success rate and accuracy greater than 99\%, as judged by random regenotyping of $10 \%$ of the samples in the subject group.
2.4. Statistical Analysis. The Hardy-Weinberg equilibrium (HWE) for each SNP was tested with a standard observedexpected chi-square test ( $\chi^{2}$ test). The overall association study was adjusted for age and gender with binary logistic regression, whereas gender-stratified analysis was adjusted for age. The genetic association analysis was carried out by constructing $2 \times 3$ tables of the genotype counts and $2 \times$ 2 tables of the allele counts for each SNP in both patient and control groups. Subsequently, Pearson $\chi^{2}$ statistics were calculated and $P$ values were computed by comparing the statistic to a $\chi^{2}$ distribution with 1 or 2 degrees of freedom for the allelic and genotypic tests. Odds ratio (OR) corresponding to $95 \%$ CI was used to assess the strength of association between the SNPs and susceptibility to high myopia. The significance of the pooled OR was determined by the $Z$-test. All statistical analyses were conducted using SPSS (version 17.0) software, and all results were considered to be statistically significant when $P<0.05$.

\section{Results}

All of the four SNPs were successfully genotyped, and the genotype distributions were within Hardy-Weinberg equilibrium in both patient and control groups $(P>0.05$, Table 2). Among the SNPs genotyped, rs9307551 was found to be significantly associated with high myopia. The minor allele (A) frequency (MAF) of rs9307551 was 0.445 in controls and 0.505 in patients, and the adjusted allelic $P$ value was $2.46 \times 10^{-4}$, with an adjusted odds ratio of $1.33(95 \%$ confidence interval, 1.14-1.54). The $P$ value of rs9307551 still showed significant association with $\mathrm{HM}$ after the Bonferroni correction (corrected $P=9.84 \times 10^{-4}$ ). No significant association was observed between HM and the other three SNPs $(P>0.05$, Table 2$)$.

We further investigated the association between HM and rs9307551 by using different genetic models. The genotype distribution of rs9307551 is shown in Table 3. The frequency of CC genotype was significantly higher in the controls than that in HM patients (31.2\% in controls and $23.6 \%$ in patients). The results suggested that subjects carrying rs9307551AC, rs9307551AA, and rs9307551AA + AC were more likely to suffer from HM than those carrying rs9307551CC (additive 1 model: $P=0.012$, adjusted OR [95\% CI] $=1.49$ [1.15-1.94]; additive 2 model: $P=3.37 \times 10^{-4}$, adjusted OR $[95 \% \mathrm{CI}]=1.75$ [1.28-2.38]; dominant model: $P=2.12 \times 10^{-4}$, adjusted OR $[95 \% \mathrm{CI}]=1.59$ [1.24-2.01]). In addition, the recessive model indicated that subjects bearing rs9307551AA had a 1.34fold (95\% CI: 1.04-1.73) increased likelihood of HM compared to those who harbor rs9307551AC and rs9307551CC. 


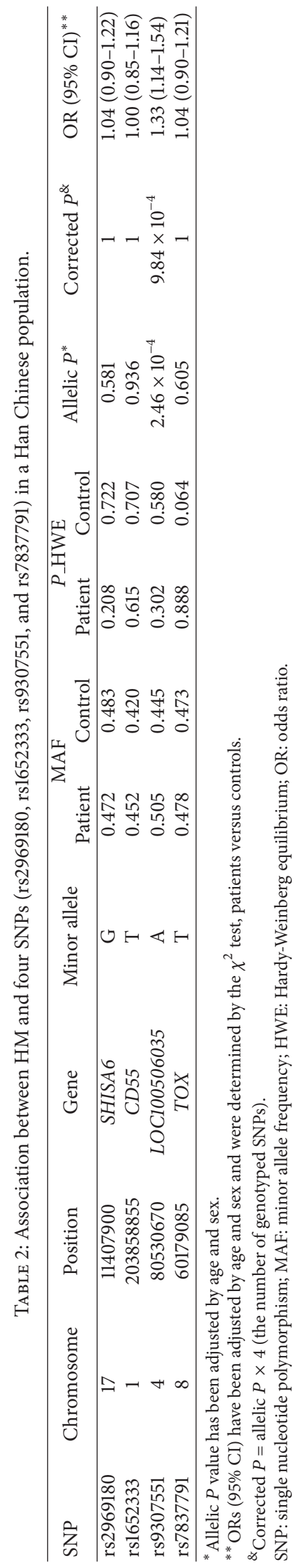


TABLE 3: The SNP of rs9307551 in the LOC100506035 gene was associated with HM in this study.

\begin{tabular}{|c|c|c|c|c|c|c|c|c|}
\hline \multirow{2}{*}{ Group } & \multicolumn{3}{|c|}{ Genotype $(n \%)$} & \multirow{2}{*}{ P_HWE } & \multirow{2}{*}{ Model } & \multirow{2}{*}{ Crude OR (95\% CI) } & \multirow{2}{*}{ Adjusted OR (95\% CI) } & \multirow{2}{*}{ Adjusted $P$} \\
\hline & AA & $\mathrm{A} / \mathrm{C}$ & $\mathrm{CC}$ & & & & & \\
\hline Control & $200(20.3)$ & $479(48.5)$ & $308(31.2)$ & 0.580 & & 1 & 1 & \\
\hline \multirow[t]{5}{*}{ Patient } & $206(25.7)$ & $408(50.7)$ & $190(23.6)$ & 0.302 & Allelic & $1.24(1.10-1.41)$ & $1.33(1.14-1.54)$ & $2.46 \times 10^{-4}$ \\
\hline & & & & & Additive 1 & $1.40(1.13-1.73)$ & $1.49(1.15-1.94)$ & 0.012 \\
\hline & & & & & Additive 2 & $1.54(1.20-1.98)$ & $1.75(1.28-2.38)$ & $3.37 \times 10^{-4}$ \\
\hline & & & & & Dominant & $1.44(1.18-1.76)$ & $1.59(1.24-2.01)$ & $2.12 \times 10^{-4}$ \\
\hline & & & & & Recessive & $1.24(1.00-1.52)$ & $1.34(1.04-1.73)$ & 0.025 \\
\hline
\end{tabular}

Crude ORs (95\% CI) were determined by the $\chi^{2}$ test, patients versus controls. Adjusted ORs (95\% CI) and adjusted $P$ values were obtained by adjusting for age and sex.

Genotype (AA/AC/CC) analyses were conducted for the allelic model (A compared with C), additive model 1 (AC compared with CC), additive model 2 (AA compared with CC), dominant model (AA + AC compared with CC), and the recessive model (AA compared with AC + CC).

TABLE 4: The association between HM and rs9307551 was gender-specific.

\begin{tabular}{|c|c|c|c|c|c|c|c|c|c|}
\hline \multirow{2}{*}{ Gender } & \multirow{2}{*}{ Group } & \multicolumn{3}{|c|}{ Genotype $(n \%)$} & \multirow{2}{*}{ P_HWE } & \multirow{2}{*}{ Model } & \multirow{2}{*}{ Crude OR (95\% CI) } & \multirow{2}{*}{ Adjusted OR ${ }^{\#}(95 \%$ CI $)$} & \multirow{2}{*}{ Adjusted $P^{\#}$} \\
\hline & & AA & $\mathrm{A} / \mathrm{C}$ & $\mathrm{CC}$ & & & & & \\
\hline \multirow{6}{*}{ Female } & Control & $82(20.3)$ & $191(47.3)$ & $131(32.4)$ & 0.417 & & 1 & 1 & \\
\hline & Patient & $133(26.9)$ & $259(52.4)$ & $102(20.7)$ & 0.241 & Allelic & $1.45(1.20-1.74)$ & $1.47(1.19-1.84)$ & $2.86 \times 10^{-4}$ \\
\hline & & & & & & Additive 1 & $1.74(1.27-2.40)$ & $1.66(1.28-2.45)$ & 0.012 \\
\hline & & & & & & Additive 2 & $2.08(1.43-3.04)$ & $2.12(1.39-3.24)$ & $4.65 \times 10^{-4}$ \\
\hline & & & & & & Dominant & $1.84(1.36-2.49)$ & $1.95(1.40-2.72)$ & $7.99 \times 10^{-5}$ \\
\hline & & & & & & Recessive & $1.45(1.06-1.98)$ & $1.41(1.00-2.01)$ & 0.050 \\
\hline \multirow{6}{*}{ Male } & Control & $118(20.2)$ & $288(49.4)$ & $177(30.4)$ & 0.966 & & 1 & 1 & \\
\hline & Patient & $73(23.5)$ & $149(48.1)$ & $88(28.4)$ & 0.521 & Allelic & $1.11(0.91-1.35)$ & $1.18(0.79-1.75)$ & 0.411 \\
\hline & & & & & & Additive 1 & $1.24(0.84-1.83)$ & $1.18(0.94-1.47)$ & 0.142 \\
\hline & & & & & & Additive 2 & $1.04(0.76-1.44)$ & $1.39(0.89-2.17)$ & 0.142 \\
\hline & & & & & & Dominant & $1.09(0.81-1.49)$ & $1.24(0.88-1.75)$ & 0.223 \\
\hline & & & & & & Recessive & $1.21(0.87-1.69)$ & $1.25(0.86-1.82)$ & 0.237 \\
\hline
\end{tabular}

${ }^{\#} P$ value and $\mathrm{ORs}(95 \% \mathrm{CI})$ were adjusted by age.

In the meantime, different genetic models were also applied to analyze the association between rs2969180, rs1652333, and rs7837791 and HM. Still, no significant association was found (data not shown).

In order to study whether rs9307551 showed significant association only with one gender, we split the subjects by gender and investigated the association by using different genetic models (Table 4). Interestingly, our data indicated that the association between rs9307551 and high myopia was gender-specific. The association was only observed in females but not males. We also found that the association was not only maintained but also strengthened in the female group, despite the reduced sample size. The allelic model showed that rs9307551A was the risk allele of HM (OR $\left.[95 \% \mathrm{CI}]=1.47[1.19-1.84], P=2.86 \times 10^{-4}\right)$. Females with rs9307551AC, rs9307551AA, and rs9307551AA + AC, respectively, had a 1.66-fold (95\% CI: $1.28-2.45, P=0.012$ ), 2.12-fold (95\% CI: 1.39-3.24, $P=4.65 \times 10^{-4}$ ), and 1.95-fold (95\% CI: $\left.1.40-2.72, P=7.99 \times 10^{-5}\right)$ increased likelihood of HM compared to those females carrying rs9307551CC. Furthermore, the recessive model showed also that female subjects bearing rs9307551AA had a 1.41-fold (95\% CI: 1.002.01) increased likelihood of HM compared to those females with rs9307551AC and rs9307551CC. None of these effects, however, was observed in males.

\section{Discussion}

In 2013, the Consortium of Refractive Error and Myopia (CREAM) presented the results from the largest international genome-wide meta-analysis and reported 24 new loci associated with myopia in the Caucasian populations, including rs2969180, rs1652333, rs9307551, and rs7837791 [14]. As shown in the results of the CREAM study [14], the "A" allele of rs9307551 showed decreasing effect with spherical equivalent. In this study, our results demonstrated that the "A" allele of rs9307551 had an increased risk of high myopia (Tables 2 and 3 ). And taking the results of these two studies together, we concluded that the direction of the effect was the same in CREAM as in our study. However, no significant association was found in the other three SNPs in this study. The sample size in this study was much smaller than that in CREAM study. This might be one of the reasons why we cannot replicate the results of CREAM. And other reasons could be allele frequency differences, different LD patterns between populations, and ethnic differences. 
On the other hand, gender-specific genetic effects have been observed in other traits $[15,16]$. In this study, our results suggested that the genetic variant located at $33 \mathrm{~kb} 5^{\prime}$ of LOC100506035 gene was associated with HM in a genderspecific way. This association was only found in females but not in males. However, there have been too few studies addressing the effect of gender on the risk of HM. In 2002, Saw et al. pointed out that myopia was associated with being male in Singaporean Chinese children [17]. But, in 2012, You et al. noted that myopia in school children in Greater Beijing was associated with female gender [18]. As a result, the gender-specific association seen in our study could provide insights into the understanding of the etiology of $\mathrm{HM}$ in the future. Gender-specific associations might result from gene-gene or gene-environment interactions. It is possible that women are exposed to an environment which interacts with the gene. These results underlie the potential importance of analyzing HM data both with and without gender as a stratifying factor.

LOC100506035 gene has also been called long intergenic nonprotein coding RNA 989 (LINC00989). Large intergenic noncoding RNAs (lincRNAs) have recently been identified as a kind of new RNA molecule in gene regulation [19, 20]. The lincRNAs, which proved functional, showed diverse biological activities, such as involvement in $\mathrm{X}$ chromosome inactivation and regulation of gene expression in cancer cells, stem cells, and development [21-24]. More than 30\% of lincRNAs were estimated to be associated with chromatinmodifying complexes, such as co-REST and PRC2, and subsequently target those complexes to specific genomic regions [19]. HOTAIR, a lincRNA which is part of the Hox gene cluster, proved to be related to breast cancer progression [25]. And ANRIL, a lincRNA regulating CDKN2A and CDKN2B, was reported to be associated with atherosclerosis [26]. However, despite the potentially broad functional impact of lincRNAs in regulation of gene expression, no lincRNAs have been reported to be related to HM. In this study, the data showed that a genetic variant of a lincRNA gene may, at least in part, play a crucial role in the development of HM in Han Chinese population. This may facilitate the study of lincRNAs and provide a new direction to investigate the genetic mechanism implicated in HM.

In summary, our data revealed the gender-specific association between rs9307551 and HM in Han Chinese population. Female carriers of rs 9307551 risk allele (A) were more likely to suffer from HM. Our results suggested the potential importance of analyzing HM data both with and without gender as a stratifying factor. In addition, our results suggested that a genetic variant in a lincRNA gene (LOC100506035) increased susceptibility to high myopia in Han Chinese.

\section{Disclaimer}

All authors had full access to the data and take full responsibility for its integrity.

\section{Conflict of Interests}

The authors declare that there is no conflict of interests regarding the publication of this paper.

\section{Acknowledgments}

The authors thank the patients and their families for their participation. This work was supported by the National Basic Research Program of China (973 Program, 2011CB504604 to Zhenglin Yang), the National Science Foundation of China [81170882 to Yi Shi; 81170883 to Zhenglin Yang; 81371048 to Bo Gong], the Department of Science and Technology of Sichuan Province, China [2012JQ0023 and 2014JZ0004 to Yi Shi], and the Department of Sichuan Provincial Health [130167 to Bo Gong].

\section{References}

[1] Y. Tano, "Pathologic myopia: where are we now?" The American Journal of Ophthalmology, vol. 134, no. 5, pp. 645-660, 2002.

[2] S. Vitale, L. Ellwein, M. F. Cotch, F. L. Ferris III, and R. Sperduto, "Prevalence of refractive error in the United States, 1999-2004," Archives of Ophthalmology, vol. 126, no. 8, pp. 1111-1119, 2008.

[3] J. H. Kempen, P. Mitchell, K. E. Lee et al., "The prevalence of refractive errors among adults in the United States, Western Europe, and Australia," Archives of Ophthalmology, vol. 122, no. 4, pp. 495-505, 2004.

[4] A. Sawada, A. Tomidokoro, M. Araie, A. Iwase, and T. Yamamoto, "Refractive errors in an elderly Japanese population: the Tajimi study," Ophthalmology, vol. 115, no. 2, pp. 363-370, 2008.

[5] M. He, Y. Zheng, and F. Xiang, "Prevalence of myopia in urban and rural children in mainland china," Optometry and Vision Science, vol. 86, no. 1, pp. 40-44, 2009.

[6] P. J. Foster, J. Hee, J. M. Tielsch, G. J. Johnson, and S. K. L. Seah, "Prevalence and risk factors for refractive errors in adult Chinese in Singapore," Investigative Ophthalmology and Visual Science, vol. 41, no. 9, pp. 2486-2494, 2000.

[7] L. Xu, J. Li, T. Cui et al., "Refractive error in urban and rural adult Chinese in Beijing," Ophthalmology, vol. 112, no. 10, pp. 1676-1683, 2005.

[8] B. J. Curtin and D. B. Karlin, "Axial length measurements and fundus changes of the myopic eye," American Journal of Ophthalmology, vol. 71, no. 1, pp. 42-53, 1971.

[9] N. A. McBrien and A. Gentle, "Role of the sclera in the development and pathological complications of myopia," Progress in Retinal and Eye Research, vol. 22, no. 3, pp. 307-338, 2003.

[10] S. M. Saw, G. Gazzard, E. C. Shin-Yen, and W. H. Chua, "Myopia and associated pathological complications," Ophthalmic and Physiological Optics, vol. 25, no. 5, pp. 381-391, 2005.

[11] M. He, J. Zeng, Y. Liu, J. Xu, G. P. Pokharel, and L. B. Ellwein, "Refractive error and visual impairment in urban children in southern China," Investigative Ophthalmology and Visual Science, vol. 45, no. 3, pp. 793-799, 2004.

[12] M. Dirani, M. Chamberlain, S. N. Shekar et al., "Heritability of refractive error and ocular biometrics: the genes in myopia (GEM) twin study," Investigative Ophthalmology \& Visual Science, vol. 47, no. 11, pp. 4756-4761, 2006.

[13] M. C. Lopes, T. Andrew, F. Carbonaro, T. D. Spector, and C. J. Hammond, "Estimating heritability and shared environmental effects for refractive error in twin and family studies," Investigative Ophthalmology and Visual Science, vol. 50, no. 1, pp. 126-131, 2009.

[14] V. J. Verhoeven, P. G. Hysi, R. Wojciechowski et al., "Genomewide meta-analyses of multiancestry cohorts identify multiple 
new susceptibility loci for refractive error and myopia," Nature Genetics, vol. 45, no. 3, pp. 314-318, 2013.

[15] R. Cáliz, L. M. Canet, C. B. Lupiañez et al., "Gender-specific effects of genetic variants within Th1 and Th17 cell-mediated immune response genes on the risk of developing rheumatoid arthritis," PLoS ONE, vol. 8, no. 8, Article ID e72732, 2013.

[16] C. Perez-Becerril, A. G. Morris, A. Mortimer, P. J. McKenna, and J. de Belleroche, "Allelic variants in the zinc transporter-3 gene, SLC30A3, a candidate gene identified from gene expression studies, show gender-specific association with schizophrenia," European Psychiatry, vol. 29, no. 3, pp. 172-178, 2014.

[17] S. M. Saw, A. Carkeet, K. S. Chia, R. A. Stone, and D. T. H. Tan, "Component dependent risk factors for ocular parameters in Singapore Chinese children," Ophthalmology, vol. 109, no. 11, pp. 2065-2071, 2002.

[18] Q. S. You, L. J. Wu, J. L. Duan et al., "Factors associated with myopia in school children in China: the Beijing Childhood Eye Study," PLoS ONE, vol. 7, no. 12, Article ID e52668, 2012.

[19] A. M. Khalil, M. Guttman, M. Huarte et al., "Many human large intergenic noncoding RNAs associate with chromatinmodifying complexes and affect gene expression," Proceedings of the National Academy of Sciences of the United States of America, vol. 106, no. 28, pp. 11667-11672, 2009.

[20] M. Guttman, I. Amit, M. Garber et al., "Chromatin signature reveals over a thousand highly conserved large non-coding RNAs in mammals," Nature, vol. 458, no. 7235 , pp. 223-227, 2009.

[21] M. E. Talkowski, C. Ernst, A. Heilbut et al., "Next-generation sequencing strategies enable routine detection of balanced chromosome rearrangements for clinical diagnostics and genetic research," American Journal of Human Genetics, vol. 88, no. 4, pp. 469-481, 2011.

[22] M. Huarte, M. Guttman, D. Feldser et al., "A large intergenic noncoding RNA induced by p53 mediates global gene repression in the p53 response," Cell, vol. 142, no. 3, pp. 409-419, 2010.

[23] S. Loewer, M. N. Cabili, M. Guttman et al., "Large intergenic non-coding RNA-RoR modulates reprogramming of human induced pluripotent stem cells," Nature Genetics, vol. 42, no. 12, pp. 1113-1117, 2010.

[24] K. C. Wang, Y. W. Yang, B. Liu et al., "A long noncoding RNA maintains active chromatin to coordinate homeotic gene expression," Nature, vol. 472, no. 7341, pp. 120-124, 2011.

[25] R. A. Gupta, N. Shah, K. C. Wang et al., "Long non-coding RNA HOTAIR reprograms chromatin state to promote cancer metastasis," Nature, vol. 464, no. 7291, pp. 1071-1076, 2010.

[26] C. E. Burd, W. R. Jeck, Y. Liu, H. K. Sanoff, Z. Wang, and N. E. Sharpless, "Expression of linear and novel circular forms of an INK4/ARF-associated non-coding RNA correlates with atherosclerosis risk," PLoS genetics, vol. 6, no. 12, Article ID e1001233, 2010. 


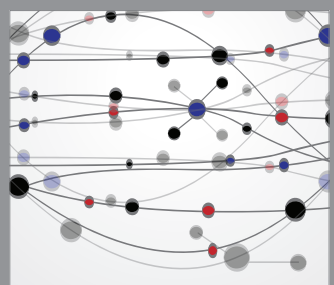

The Scientific World Journal
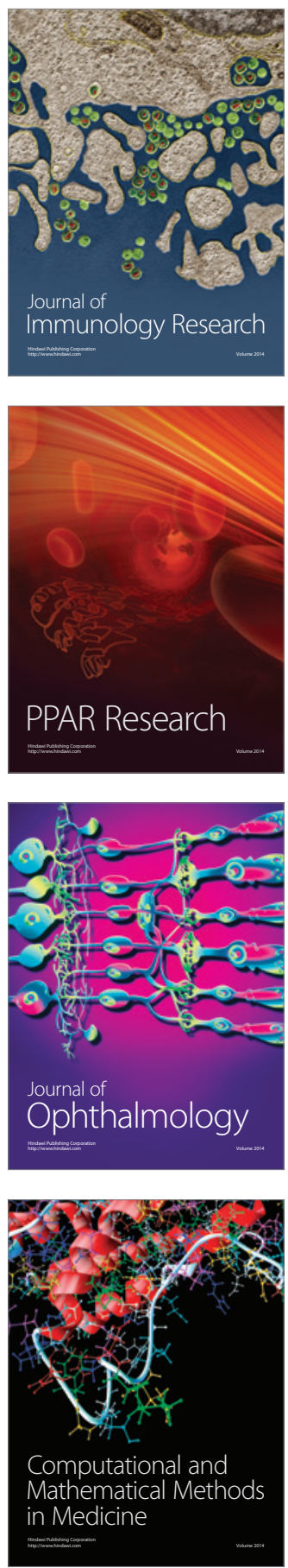

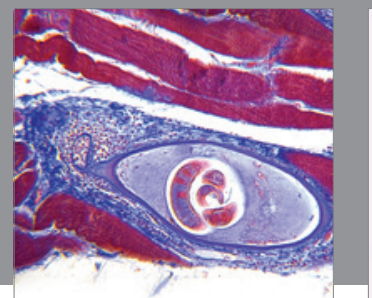

Gastroenterology

Research and Practice
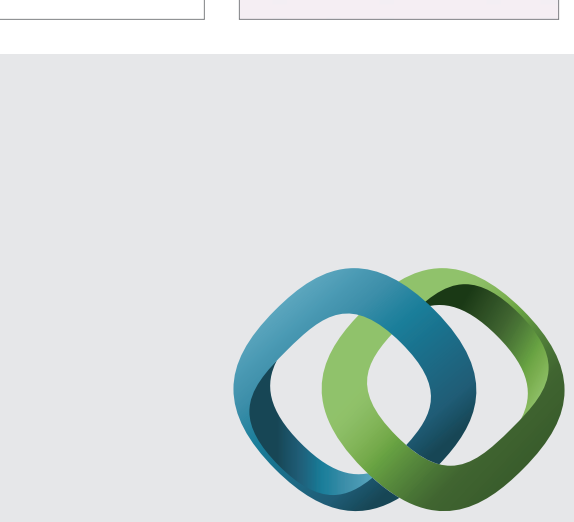

\section{Hindawi}

Submit your manuscripts at

http://www.hindawi.com
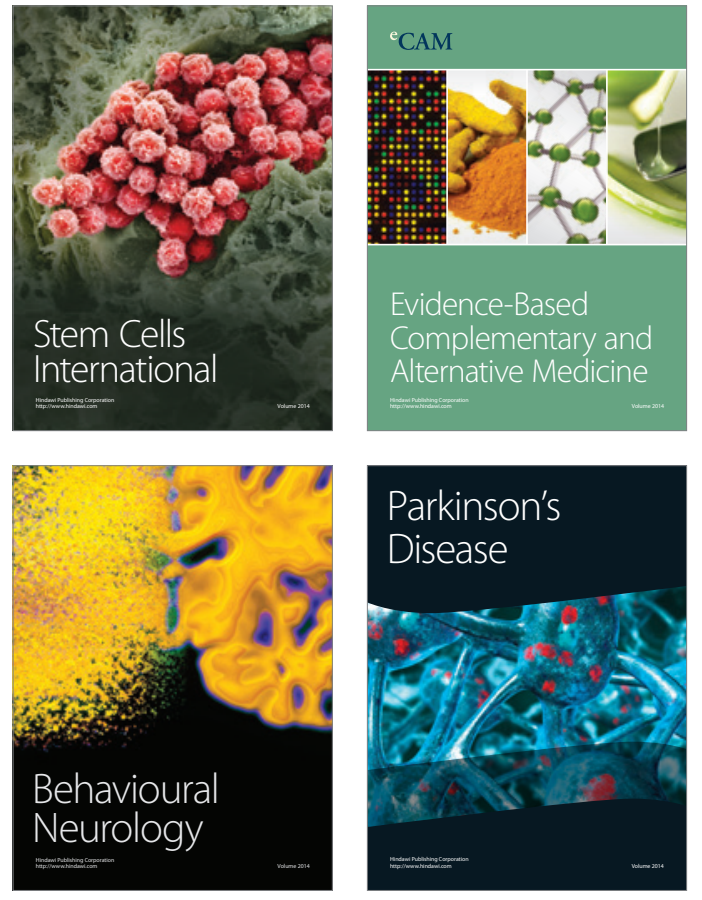
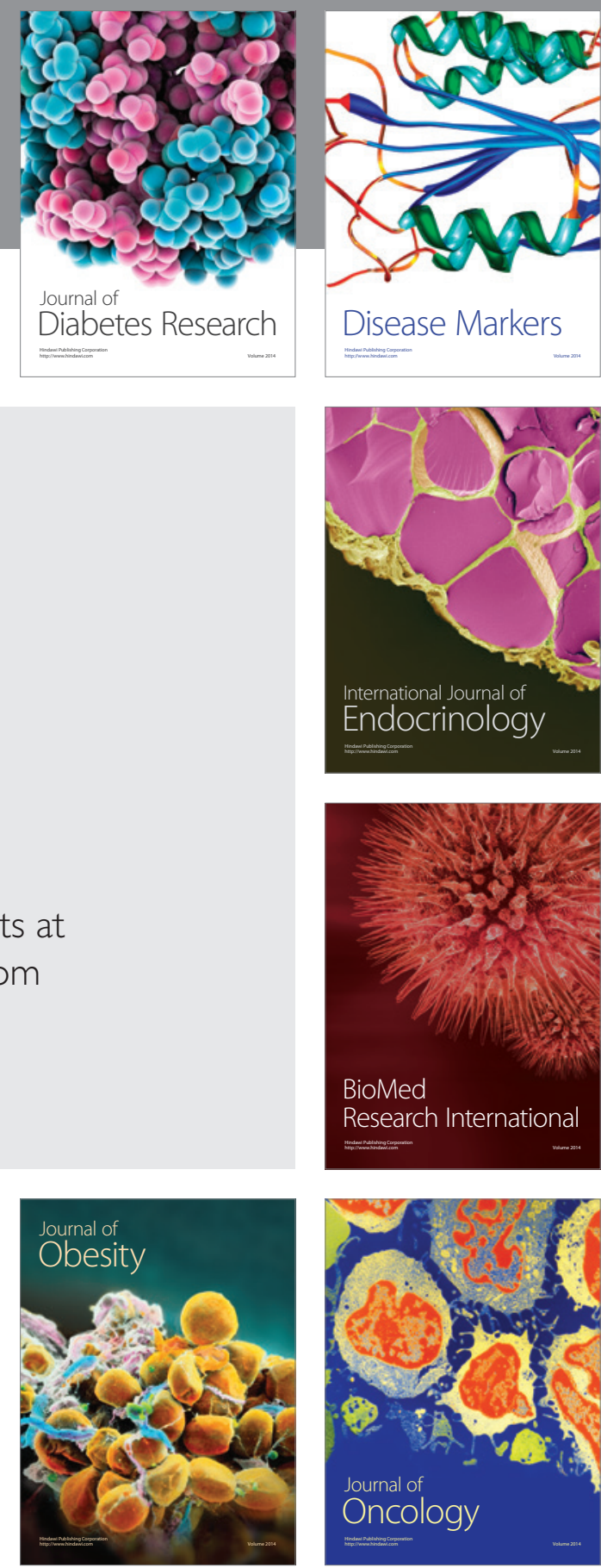

Disease Markers
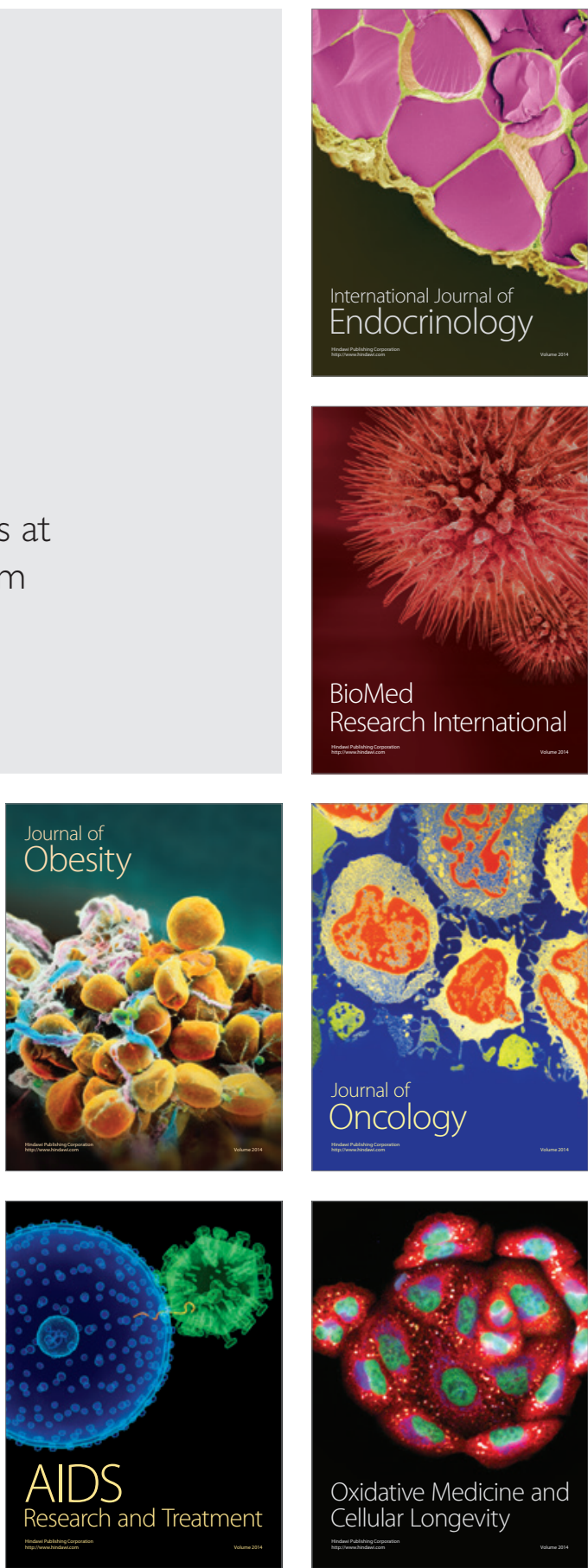